\title{
Modeling enzyme-ligand binding in drug discovery
}

\author{
Janez Konc ${ }^{1,2}$, Samo Lešnik and Dušanka Janežičč
}

\begin{abstract}
Enzymes are one of the most important groups of drug targets, and identifying possible ligand-enzyme interactions is of major importance in many drug discovery processes. Novel computational methods have been developed that can apply the information from the increasing number of resolved and available ligand-enzyme complexes to model new unknown interactions and therefore contribute to answer open questions in the field of drug discovery like the identification of unknown protein functions, off-target binding, ligand 3D homology modeling and induced-fit simulations.
\end{abstract}

Keywords: Unknown protein fuctions, Off-target binding, Drug repositioning, Ligand 3D homolgy modeling, Induced-fit simulations, ProBis-ligands web server

\section{Background}

Predicting ligands that bind with sufficient strength to a corresponding protein is a challenging task in biochemistry and has significant implication in the discovery of new drug candidates. Many approaches have been developed for this task; the most commonly used being molecular docking [1]. However one of the main drawbacks of classical template-free docking is that every molecule is docked ab initio, and no information from existing similar protein-ligand complexes is taken into consideration. Therefore alternative approaches that use information from existing protein-ligand complexes, which can be obtained from freely-available databases, such as the Protein Data Bank [2] are becoming increasingly important. The main assumption of such approaches is that similar protein binding sites bind similar ligands, and thus a known ligand from one protein can be transposed to a similar binding site in another protein that was previously not known to bind this ligand. Transposition of ligands is based on accurate alignments of three-dimensional amino-acid patterns or of their corresponding functional groups in the proteins' binding sites; due to their local nature in the binding sites, such alignments may not be possible with standard sequence or structure alignment

\footnotetext{
*Correspondence: dusanka.janezic@upr.si

2 Faculty of Mathematics, Natural Sciences and Information Technologies,

University of Primorska, Glagoljaška 8, SI-6000 Koper, Slovenia

Full list of author information is available at the end of the article
}

approaches. Ligand transposition shown in Fig. 1 can thus be a powerful approach, which can be used in pharmaceutical applications such as drug repositioning [3-6], ligand-homology modeling [7-9], induced-fit simulation [10] and binding site prediction [11-13]. Information about the software described in the following sections is available in Table 1.

\section{Function prediction}

Proteins interact with one another and with other molecules, mediate metabolic and signaling pathways and thus regulate cellular processes [14]. One of the fundamental tasks of proteins is to act as enzymes [15], i.e., biological catalysts that increase the rate of practically all chemical reactions that are taking place within cells. Due to their central role in biological function, they control mechanisms leading to healthy and diseased states in the organism. However, for a substantial number of proteins, and subsequently enzymes, their functions are not known, therefore an important challenge in structural genomics is the prediction of function of these uncharacterized proteins [16]. While experimental determination of a protein function is still the most reliable way to characterize unknown proteins, it is difficult to prioritize functional experiments among the many possible functions a protein could perform. To guide experimentalist, a number of computer approaches are routinely used to predict protein function. However, many are based on sequence

\section{Chemistry Central}

(c) 2015 Konc et al. This article is distributed under the terms of the Creative Commons Attribution 4.0 International License (http://creativecommons.org/licenses/by/4.0/), which permits unrestricted use, distribution, and reproduction in any medium, provided you give appropriate credit to the original author(s) and the source, provide a link to the Creative Commons license, and indicate if changes were made. The Creative Commons Public Domain Dedication waiver (http://creativecommons.org/ publicdomain/zero/1.0/) applies to the data made available in this article, unless otherwise stated. 


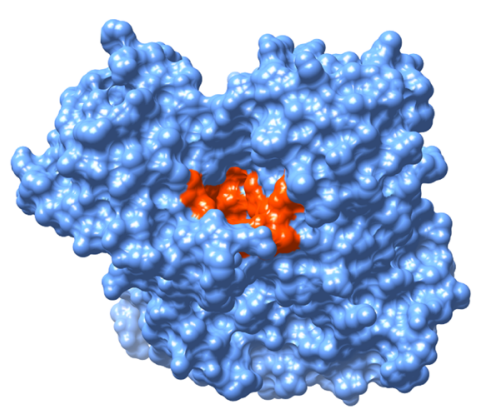

Enzyme binding site

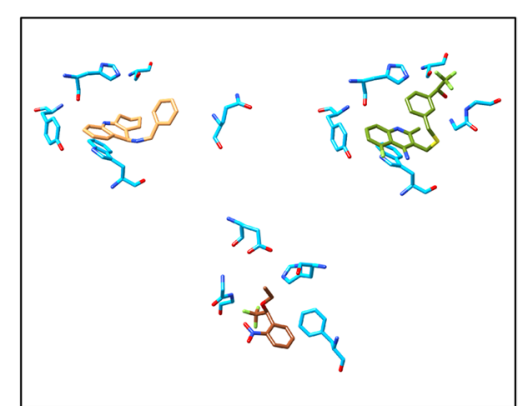

PDB ligands and binding sites

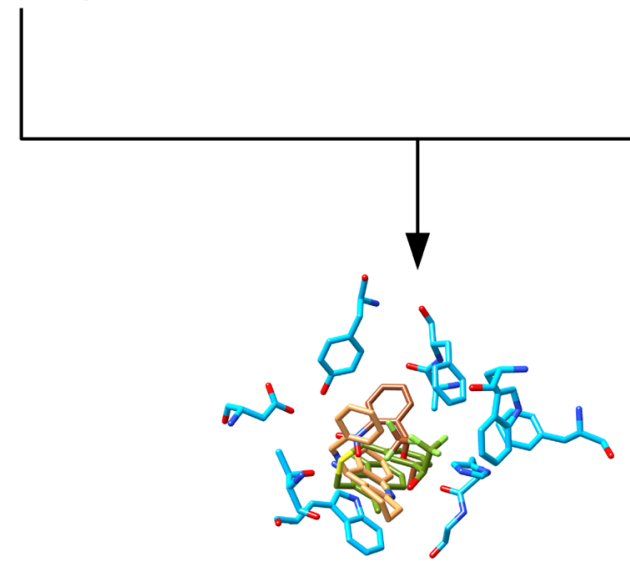

Ligands transposed to enzyme binding site

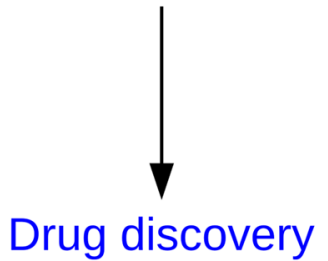

Fig. 1 Flowchart of binding site comparison with subsequent ligand transposition

and overall structural homology [17, 18], consequently often missing similarities when only local binding sites are conserved. The identification of such binding sites on the protein surface is therefore usually the starting point for protein function annotation. Moreover, because knowledge of the binding site location is the prerequisite for molecular docking, binding site identification is often a first step in structure-based drug design.

Based on the idea that proteins with similar local binding sites perform similar function, an algorithm ProBiS and its corresponding web server $[19,20]$ was developed. ProBiS uses a fast maximum clique algorithm [21] to compare a query protein to members of a database of 3D protein structures and detects with sub-residue precision structurally similar binding sites as patterns of physiochemical properties on the protein surface. The algorithm thus identifies database proteins that share local structural similarities with the query protein independent of the global protein folds, and generates structure-based alignments for every query protein-database protein pair. Moreover, structural similarity scores are calculated for the query protein surface residues, and are expressed as different colors on the protein's surface. ProBiS was tested for its binding site detection ability on a set of 39 protein structures and by comparing it to an evolutionary conservation mapping method ConSurf [22] and an energybased method Q-SiteFinder [23], it was shown that ProBiS outperformed both of the two aforementioned methods. Moreover, to demonstrate ProBiS' unique ability to detect and align similar binding sites in the absence of global fold similarity, the authors examined 10 pairs of protein structures, where the two members of each pair 
Table 1 Software packages for modeling protein-ligand binding using ligand transposition

\begin{tabular}{|c|c|c|c|}
\hline Name & URL & Description & Availability \\
\hline ProBis & http://www.probis.cmm.ki.si/ & $\begin{array}{l}\text { Detects structurally similar binding sites } \\
\text { without reference to known binding sites }\end{array}$ & Freely-accessible web server \\
\hline GalaxySite & http://www.galaxy.seoklab.org/site/ & $\begin{array}{l}\text { Combines binding site information from } \\
\text { known proteins with molecular docking } \\
\text { to predict ligand binding amino acid } \\
\text { residues }\end{array}$ & Freely-accessible web server \\
\hline Surflex-PSIM & http://www.biopharmics.com/downloads.html & $\begin{array}{l}\text { Fully automated ligand binding pocket } \\
\text { detection and comparison based on sur- } \\
\text { face similarities to other known proteins }\end{array}$ & Not freely-accessible \\
\hline POP & https://www.sites.google.com/site/offtargetpipeline/ & $\begin{array}{l}\text { Integrated computational method for } \\
\text { proteome-wide off target identification }\end{array}$ & $\begin{array}{l}\text { Free for academic users and } \\
\text { not-for-profit institutions }\end{array}$ \\
\hline FINDSITE ${ }^{\text {comb }}$ & http://www.cssb.biology.gatech.edu/FINDSITE-COMB/ & $\begin{array}{l}\text { Threading/structure-based, proteomic- } \\
\text { scale virtual ligand screening approach }\end{array}$ & $\begin{array}{l}\text { Freely-accessible for web server } \\
\text { for academic users }\end{array}$ \\
\hline ProBiS-ligands & http://www.probis.cmm.ki.si/ligands/ & $\begin{array}{l}\text { Detects and transposes ligands between } \\
\text { similar binding sites }\end{array}$ & Freely-accessible web server \\
\hline FragFEATURE & https://simtk.org/home/frag-feature/ & $\begin{array}{l}\text { A machine learning approach to predict } \\
\text { small molecules fragments preferred by } \\
\text { a target }\end{array}$ & Freely-accessible \\
\hline
\end{tabular}

exhibited different folds but had known similar binding sites and performed a similar function. ProBiS was compared to three different structural alignment algorithms; DaliLite [24], MolLoc [25] and MultiBind [26]. The comparison between the methods was made by calculating the RMSD between previously identified, similar binding site residues, after the proteins in the pair have been superimposed. ProBiS demonstrated, by far, the lowest average RMSD in comparison to other methods; while also having the ability to align binding sites in an unsupervised fashion, which allows it to perform automatic, large database searches. ProBiS, in combination with molecular dynamics, was also used to propose the function to a protein of unknown activity-the Tm1631 protein from T. maritima [27]. The binding site comparison revealed numerous similarities with nucleotide binding sites; including specifically, a DNA-binding site of endonuclease IV. Based on the superimposition of Tm1631 with endonuclease IV, a hypothetical model of the Tm1631-DNA complex was constructed. This model was validated with the use of CHARMM $[28,29]$ to perform a molecular dynamics simulation, which showed that the interactions predicted by the Tm1631-DNA model correspond well to those known to be importation in the endonuclease IV-DNA complex. The simulation also showed that the binding free energies of the model and the known complex were in close agreement. The authors thus concluded that the Tm1631 protein could be a DNA binding enzyme with endonuclease activity. ProBiS was also used to identify conserved binding sites on hemagglutinin, a protein responsible for binding the influenza virus to cells [30]. A local structural superimposition across all subtypes and strains of hemagglutinin available in the PDB at the time, revealed a new conserved region on hemagglutinin, a potential conserved target for influenza drug and vaccine development.

Another freely-accessible web server for binding site annotation-GalaxySite [31] combines binding site information from known proteins with molecular docking to predict ligand binding amino acid residues. Initially the server uses HHsearch [32] to search for similar proteinligand complexes in the structural databases. The highest ranking ligands are transposed from the target to the query structure where their binding conformation is optimized using the LigDockCSA protein-ligand docking program [33]. Binding-site residues are then identified based on their proximity to the docked ligand. GalaxySite was extensively tested on different ligand binding prediction test sets, on which it showed superior or similar performance compared to other state of the art prediction methods.

Moreover, Surflex-PSIM [34], a novel method combining initial binding site recognition with subsequent binding site comparisons has been developed. The newest version of Surflex-PSIM is able to automatically detect ligand binding pockets and compare them, based on their surface similarity to other binding sites extracted from large protein databases (e.g. PDB). The method was tested on a set of eight proteins, whose function was unknown at the time of the testing. All of the eight proteins were screened against $\sim 60,000$ ligand binding sites from the PDB. Surflex-PSIM correctly identified functional matches that predated query protein biochemical annotation for five out of the eight proteins. In addition, 
12 currently unannotated proteins were also screened, resulting in a large number of statistically significant binding site matches, which could suggest likely functions for these uncharacterized proteins. Surflex-PSIM was also used as a part of a combined computational approach which identified known PPAR $\alpha$ agonists as also being cyclooxygenase (COX) inhibitors [35]. Pockets of $9 \mathrm{COX}$ enzymes were compared to 14 human PPAR $\alpha$ binding pockets and the method identified high similarity of pocket surfaces between proteins with the PDB codes 2rew (PPAR $\alpha$ receptor) and 3rr3 (COX-2 enzyme). Subsequent experiments confirmed that fenofibric acid, a known PPAR $\alpha$ agonist, does in fact inhibit, in a does dependent manner, both the COX-1 and COX-2 enzymes.

\section{Drug repositioning}

Drug repositioning or repurposing is a principle of discovering novel therapeutic indications for existing approved drugs, which provides an alternative and costefficient strategy of discovering disease therapeutics [36]. A prerequisite for drug repurposing is drug promiscuity (polypharmacology), which is a drug's ability to bind to several different targets. A recent study suggests that the most important factor contributing to the observed promiscuity of many drugs is the local binding site similarities between different protein targets [3]. It was discovered that off-target binding is the major cause of unwanted side-effects for many drugs from a wide range of therapeutic areas [37]. Therefore binding site comparison methods may have an important role in identifying the polypharmacological activity of molecules.

Recently, an integrated computational method for proteome-wide off target identification, abbreviated POP (proteome-wide off-target pipeline) was developed. POP combines ligand binding site comparison analysis, protein-ligand docking and electrostatic potential calculation to identify possible promiscuous protein-ligand interactions throughout the proteasome. The core component of this method is the well-established software for binding site comparison SMAP [38, 39]. SMAP initially detects the location and boundary of the query protein ligand binding site. The binding site is then compared against target $3 \mathrm{D}$ protein structures using a sequenceorder independent profile-profile alignment (SOIPPA) algorithm [39] that is able to detect similar binding sites between structurally unrelated proteins. The next step is the superimposition (and subsequent ligand transposition) of the query binding site to the top scoring target binding sites. The transposed ligand binding pose serves as the starting structure in the following docking and scoring. The highest scoring protein-ligand complexes reflect the possible off-targets of the ligand. In addition, the electrostatic potential binding energy and similarity between the binding sites can be calculated based on the binding pose of the ligand. POP was applied to identify possible off-targets of the HIV protease inhibitor nelfinavir. The protein-ligand complex of HIV protease bound with nelfinavir (PDB code: 1ohr) was searched against a variety of human protein structures and models. Top ranked hits contained multiple members of the protein kinase superfamily, most of which are on the upstream of the Akt1 and Akt2 enzymes in the AKT pathway [40], which is in correlation with the experimentally observed anti-cancer effect of nelfinavir.

\section{Ligand 3D homology modeling}

Virtual screening is a widely used approach in the early stage of pharmaceutical discovery [41]. In practice, we distinguish two broad categories of virtual screenings: (a) ligand based and (b) structure based [42, 43]. Ligand based virtual screening is relatively fast, however the method requires previously known ligands that bind to a certain target; this hinders its universality and large-scale application. On the other hand, structure based virtual screening uses the structure of a target/target binding site, to which it docks potential drug molecules and evaluates the binding likelihood using different scoring functions; its main advantage is that no prior knowledge of known active ligands is needed. The main disadvantage of structure based virtual screening is the requirement for high-resolution structures of target proteins, which are in many cases not available, especially for G-protein coupled receptors and ion-channels [44]. However, with increasing number of holo protein structures deposited in large protein databases, a novel type of virtual screening, termed ligand-homology modeling (LHM) is gaining recognition [7-9, 45]. LHM is a knowledge-based approach that relies on the fact that evolutionary related proteins share similar functions and thus bind similar ligands, and that this information can be used to predict ligand-target interactions. In general, LHM algorithms start with identifying and aligning similar binding sites between a query and target database holo proteins, which is then followed by transposing the ligands from target proteins to the query binding site.

One of the main shortcomings of the LHM is the need for sufficient numbers of holo proteins that share similar binding sites to the query protein. To overcome this serious disadvantage, a novel combined ligand homology modeling approach, FINDSITE ${ }^{\text {comb }}$ was developed $[46,47]$. FINDSITE ${ }^{\text {comb }}$ is a composite approach consisting of the FINDSITE ${ }^{\text {filt }}$ and the FINSDSITE ${ }^{\mathrm{X}}$ algorithms. While the former still uses known holo proteins deposited in protein databases, FINSDSITE ${ }^{\mathrm{X}}$ circumvents this requirement by employing homology modeling to model 
structures of target proteins not yet available in protein databases. The FINDSITE ${ }^{\text {filt }}$ algorithm works by initially finding template holo protein structures that are evolutionary related to the target structure from the PDB database. Next, a heuristic structure-pocket alignment method is used to superimpose template pockets to the target structure and a sequence dependent scoring function to rank the best templates (based on the structurepocket alignment) from whose their corresponding ligands will be used as reference small molecules in subsequent ligand based virtual screening. FINDSITE ${ }^{\mathrm{X}}$ on the other hand, as mentioned, employs homology modeling methods to model structures of template proteins, which are then aligned and compared to target proteins using a global structure alignment method. Similar as in FINDSITE ${ }^{\text {filt }}$, the ligands of the top ranked templates are used as reference small molecules for ligand based virtual screening, where the appropriate template ligands are obtained from the DrugBank [48] and ChEMBL [49] databases. By combining the scores obtained from FINDSITE $^{\text {filt }}$ and FINDSITE ${ }^{\mathrm{X}}$ ligand based virtual screening runs, the method evaluates the likelihood of each compound of being a true active. The performance of FINDSITE ${ }^{\text {comb }}$ was thoroughly evaluated both on the directory of useful decoys (DUD) database [50] and experimentally on eight different protein targets [51]. In the former evaluation, FINDSITE ${ }^{\text {comb }}$ outperformed, in most cases, established docking methods, such as AUTODOCK Vina [52] and DOCK 6 [53]; demonstrating significantly higher enrichment factors and areas under the ROC curves. Testing FINDSITE ${ }^{\text {comb }}$ on eight different proteins with subsequent experimental ligand binding evaluations, the authors identified 47 , mostly novel small molecules with $\mu \mathrm{M}$ or better affinities. Of those, 10 ligands showed affinities in the nanomolar range (for dihydropholate reductase from E.coli and two mammalian protein tyrosine phosphatases).

Also a recently developed, freely-available web server, which enables ligand homology modeling, is ProBiSligands (Fig. 2) [45], a method that uses a fast maximum clique algorithm [21] to screen the non-redundant PDB database, to find structurally similar target binding sites to user provided input query protein, independent of their sequence or global structural similarity. Ligands which are bound in the identified similar binding sites are transposed to the query protein by rotation and translation of their atoms' coordinates generated by the superimposition matrices acquired from the initial superposition of the query and target proteins. ProBiSligands is able to predict protein-protein, protein-small molecules, protein-nucleic acid, and protein-ion interactions. The performance of the web server was assessed with a test set [54] containing 500 proteins models and their corresponding experimental structures. The success of ligand prediction was measured by calculating the correspondence between the predicted ligand binding sites, i.e. query residues $<4 \AA$ from the first cluster of predicted small molecules or ions and the actual known binding sites for each of the 500 proteins. This binding site prediction results were evaluated with the Matthews correlation coefficient, precision and recall (for definitions of each see, e.g. [54]). Moreover to assess the similarity of predicted ligands with the actual ones, each highest scoring (by Z-score) predicted ligand from the first small molecule or ion cluster was compared with the actual known ligands using an in-house developed 2D molecular graph matching algorithm. Ligand similarities were expressed as the Tanimoto coefficients and were averaged across all comparisons. ProBiS-ligands showed encouraging results, with the average ligand similarity Tanimoto coefficient of 0.61 (0.55) and MCC of 0.54 (0.41) (the values in parenthesis are for the modeled structure). Even when all templates sharing $>10 \%$ sequence identity with the target were removed (to simulate the lack of similar templates that frequently occur in real world simulations), reasonable predictions for the experimental protein structures were still possible with the average ligand similarity of 0.40 and MCC of 0.28 . In contrary, for protein models, templates of at least $20-30 \%$ sequence identity were required to obtain similar accuracy.

As discussed, an important drawback in classical screening methods is that large molecular databases (usually containing millions of compounds) have to be screened to find potential drug candidates. Given this difficulty of thoroughly exploring the chemical-space of drug like molecules, fragment-based approaches have emerged. Fragments refer to low-molecular-weight molecules, usually 140-300 Daltons in weight $[55,56]$, that can be connected to form larger molecules. It was shown that fragments have higher hit rates compared to large, complex drug-like molecules because they are less likely to possess suboptimal interactions or physical clashes with the target [57]. Moreover, a fragment library can provide a more compact and tractable basis set for chemical space than standard small molecule libraries [58]. A novel knowledge-based fragment binding predictor FragFEATURE was developed that overcomes many limitations of the existing fragment binding predictors [59]. Using information from the PDB, the authors preliminarily created a database linking local protein structural environments to the small molecule fragments they bind. Given the structural environments from a target protein, FragFEATURE compares them to the database to find similar structural environments and identifies statistically preferred fragments. The results demonstrated the method's ability to rediscover fragments corresponding 


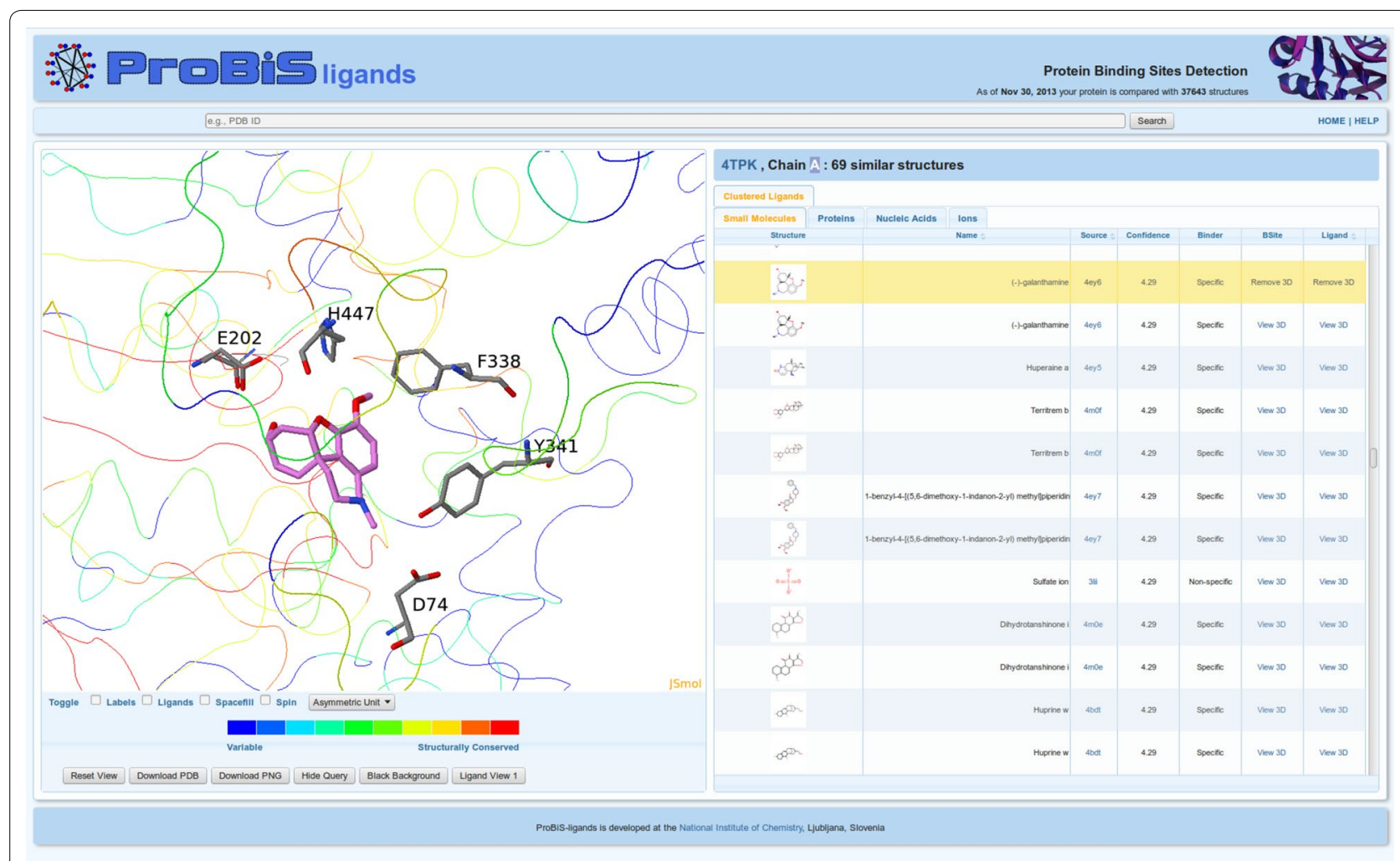

Fig. 2 Ligand homology modeling using ProBiS-ligands web server on the example of butyrylcholinesterase enzyme (PDB code: 4tpk). On the right side of the screen is the list of predicted ligands with their corresponding Z-scores, specificities and PDB codes of protein structures from which they were transposed. The selected ligand's row is highlighted orange. On the left side is the Jsmol viewer that contains the three-dimensional pose of the selected predicted ligand (galantamine, sticks, violet) and the predicted binding amino-acid residues (sticks, CPK colors, black labels)

to the ligands bound with $74 \%$ precision and $82 \%$ recalls on average. For many protein targets, FragFEATURE was able to identify high scoring fragments that are substructures to known inhibitors. Such predicted fragments can serve as inputs to fragment-based drug design or serve as refinement criteria for creating target-specific compound libraries for experimental or computational screening.

\section{Induced-fit simulation}

One of the main downsides of many established molecular docking algorithms is their inability to consider binding site flexibility [60]. This can present a serious drawback, especially when only a crystal structure without its corresponding ligands (apo structure) is available. Such a protein can be locked in a conformation that is a poor representation of an actual spatial arrangement of binding site amino acid residues upon ligand binding [61], making it a poor candidate for target based virtual screening studies.

To overcome difficulties where the binding pocket conformation is unsuitable for docking, our group developed a new simple and fast approach to simulate the induced-fit conformational changes of protein structures upon binding. For example, the UDP-N-acetylglucosamine enolpyruvyl transferase enzyle enzyme (MurA, PDB code: 1uae) from $E$. coli exists in the PDB only as apoprotein and our initial docking study with the docking program FRED software [62] showed that most druglike molecules were unable to dock in this binding site without exhibiting severe clashes with the amino acid residues. To circumvent this problem, ProBiS-ligands [45] was used in combination with standard molecular minimization algorithms. With the E. coli MurA crystal structural as input, ProBiS-ligands enabled the identification and transposition of a ligand (PDB ligand code: TAV) bound originally in a binding site of the MurA enzyme from $E$. cloace (PDB code: $1 \mathrm{ybg}$ ). With the newfound ligand transposed to the query binding site and all the original ligands and water molecules removed, molecular minimization was performed on this complex to obtain an open binding site structure of the enzyme that was more suitable for docking. The docking was then repeated on this opened binding site against the ZINC Drugs Now database [63], which yielded very 
good inhibitors of MurA enzyme as three of the highest scoring compounds that were biochemically tested for their inhibitory ability showed lower than $1 \mu \mathrm{M} \mathrm{IC} \mathrm{I}_{50}$ values and are now subject for further optimization and research (in preparation).

\section{Conclusion}

Ever increasing numbers of 3D holo enzyme structures deposited in large protein databases enable that the information of known enzyme-ligand interactions be used in predicting and evaluating novel complexes-a key step in drug discovery. Knowledge-based computational methods that apply this information have been successfully used in a wide variety of fields that are of interest to pharmaceutical research; from drug repositioning to simulating induced-fit upon ligand binding. Increased usage of knowledge-based methods for modeling ligand-enzyme interactions is expected in the near future, especially in the early stages of drug discovery processes.

\section{Authors' contributions}

JK, SL, DJ worte the manuscript. All authors read and approved the final manuscrpit.

\section{Author details \\ ${ }^{1}$ National Institute of Chemistry, Hajdrihova 19, SI-1000 Ljubljana, Slovenia. \\ 2 Faculty of Mathematics, Natural Sciences and Information Technologies, \\ University of Primorska, Glagoljaška 8, SI-6000 Koper, Slovenia.}

\section{Acknowledgements}

Financial support through Grants P1-0002, J1-6743 and L1-6745 of the Ministry of Higher Education, Science and Technology of Slovenia and Slovenian Research Agency is acknowledged.

\section{Compliance with ethical guidelines}

\section{Competing interests}

The authors declare that they have no competing interests.

Received: 21 July 2015 Accepted: 29 September 2015

Published online: 06 October 2015

\section{References}

1. Yuriev E, Ramsland PA (2013) Latest developments in molecular docking: 2010-2011 in review. J Mol Recognit 26:215-239

2. Rose PW et al (2011) The RCSB Protein Data Bank: redesigned web site and web services. Nucleic Acids Res 39:D392-D401

3. Haupt VJ et al (2013) Drug promiscuity in PDB: protein binding site similarity is key. PLoS one 8:e65894

4. Škedelj V et al (2012) 6-Arylpyrido[2,3-d]pyrimidines as novel ATPcompetitive inhibitors of bacterial D-alanine:D-alanine ligase. PLoS One 7:e39922

5. Strittmatter SM (2014) Overcoming drug development bottlenecks with repurposing: old drugs learn new tricks. Nat Med 20:590-591

6. Sethi G et al (2015) Multiscale Modelling of relationships between protein classes and drug behavior across all diseases using the CANDO platform. Mini Rev Med Chem 15:705-717

7. Brylinski M, Skolnick J (2009) FINDSITELHM: a threading-based approach to ligand homology modeling. PLoS Comput Biol 5:e1000405

8. Moriaud F et al (2009) Computational fragment-based approach at PDB scale by protein local similarity. J Chem Inf Model 49:280-294
9. Lee HS, Im W (2012) Identification of ligand templates using local structure alignment for structure-based drug design. J Chem Inf Model 52:2784-2795

10. Osguthorpe DJ et al (2012) Exploring protein flexibility: incorporating structural ensembles from crystal structures and simulation into virtual screening protocols. J Phys Chem B 116:6952-6959

11. Wass MN et al (2010) 3DLigandSite: predicting ligand-binding sites using similar structures. Nucleic Acids Res. doi:10.1093/nar/gkq406

12. Roche DB et al (2011) FunFOLD: an improved automated method for the prediction of ligand binding residues using 3D models of proteins. BMC Bioinform 12:160

13. Roche DB et al (2013) The FunFOLD2 server for the prediction of proteinligand interactions. Nucleic Acids Res 41:W303-W307

14. Gonzalez MW, Kann MG (2012) Chapter 4: protein interactions and disease. PLoS Comput Biol 8:e1002819

15. Cooper GM (2000) The central role of enzymes as biological catalysts. http://www.ncbi.n/m.nih.gov/books/NBK9921/

16. Konc J, Janežič D (2014) Binding site comparison for function prediction and pharmaceutical discovery. Curr Opin Struct Biol 25:34-39

17. Radivojac P et al (2013) A large-scale evaluation of computational protein function prediction. Nat Methods 10:221-227

18. Petrey D et al (2015) Template-based prediction of protein function. Curr Opin Struct Biol 32:33-38

19. Konc J, Janežič D (2010) ProBiS algorithm for detection of structurally similar protein binding sites by local structural alignment. Bioinformatics 26:1160-1168

20. Konc J, Janežič D (2010) ProBiS: a web server for detection of structurally similar protein binding sites. Nucleic Acids Res 38:W436-W440

21. Konc J, Janezic D (2007) An improved branch and bound algorithm for the maximum clique problem. MATCH Commun Math Comput Chem 58:569-590

22. Glaser $\mathrm{F}$ et al (2003) ConSurf: identification of functional regions in proteins by surface-mapping of phylogenetic information. Bioinformatics 19:163-164

23. Laurie ATR, Jackson RM (2005) Q-SiteFinder: an energy-based method for the prediction of protein-ligand binding sites. Bioinformatics 21:1908-1916

24. Holm L, Park J (2000) DaliLite workbench for protein structure comparison. Bioinformatics 16:566-567

25. Angaran S et al (2009) MolLoc: a web tool for the local structural alignment of molecular surfaces. Nucleic Acids Res. doi:10.1093/nar/gkp405

26. Shulman-Peleg A et al (2008) MultiBind and MAPPIS: webservers for multiple alignment of protein 3D-binding sites and their interactions. Nucleic Acids Res 36:W260-W264

27. Konc $J$ et al (2013) Structure-based function prediction of uncharacterized protein using binding sites comparison. PLoS Comput Biol 9:e1003341

28. Brooks BR et al (2009) CHARMM: the biomolecular simulation program. J Comput Chem 30:1545-1614

29. Miller BT et al (2008) CHARMMing: a new, flexible web portal for CHARMM. J Chem Inf Model 48:1920-1929

30. Yusuf M et al (2013) Structurally conserved binding sites of hemagglutinin as targets for influenza drug and vaccine development. J Chem Inf Model 53:2423-2436

31. Heo L et al (2014) GalaxySite: ligand-binding-site prediction by using molecular docking. Nucleic Acids Res 42:W210-W214

32. Söding J (2005) Protein homology detection by HMM-HMM comparison. Bioinformatics 21:951-960

33. Shin W-H et al (2011) LigDockCSA: protein-ligand docking using conformational space annealing. J Comput Chem 32:3226-3232

34. Spitzer $R$ et al (2014) Protein function annotation by local binding site surface similarity. Proteins Struct Funct Bioinforma 82:679-694

35. Cleves AE, Jain AN (2014) Chemical and protein structural basis for biological crosstalk between PPAR $\alpha$ and COX enzymes. J Comput Aided Mol Des 29:101-112

36. Haupt VJ, Schroeder M (2011) Old friends in new quise: repositioning of known drugs with structural bioinformatics. Bioinform, Brief. doi:10.1093/ bib/bbr011

37. Xie L et al (2012) Novel computational approaches to polypharmacology as a means to define responses to individual drugs. Annu Rev Pharmacol Toxicol 52:361-379 
38. Xie L, Bourne PE (2007) A robust and efficient algorithm for the shape description of protein structures and its application in predicting ligand binding sites. BMC Bioinform 8:S9

39. Xie L, Bourne PE (2008) Detecting evolutionary relationships across existing fold space, using sequence order-independent profile-profile alignments. Proc Nat Acad Sci USA 105:5441-5446

40. Xie L et al (2011) Drug discovery using chemical systems biology: weak inhibition of multiple kinases may contribute to the anti-cancer effect of Nelfinavir. PLoS Comput Biol 7:e1002037

41. Chen $\mathrm{H}$ et al (2006) On evaluating molecular-docking methods for pose prediction and enrichment factors. J Chem Inf Model 46:401-415

42. Srinivas Reddy A et al (2007) Virtual screening in drug discovery-a computational perspective. Curr Protein Pept Sci 8:329-351

43. Schneider G (2010) Virtual screening: an endless staircase? Nat Rev Drug Discov 9:273-276

44. Carpenter EP et al (2008) Overcoming the challenges of membrane protein crystallography. Curr Opin Struct Biol 18:581-586

45. Konc J, Janežič D (2014) ProBiS-ligands: a web server for prediction of ligands by examination of protein binding sites. Nucleic Acids Res 42:W215-W220

46. Zhou H, Skolnick J (2013) FINDSITEcomb: a Threading/structure-based proteomic-scale virtual ligand screening approach. J Chem Inf Model $53: 230-240$

47. Zhou H, Skolnick J (2012) FINDSITEX: a structure-based, small molecule virtual screening approach with application to all identified human GPCRs. Mol Pharm 9:1775-1784

48. Wishart DS et al (2006) DrugBank: a comprehensive resource for in silico drug discovery and exploration. Nucleic Acids Res 34:D668-D672

49. Gaulton A et al (2011) ChEMBL: a large-scale bioactivity database for drug discovery. Nucleic Acids Res. doi:10.1093/nar/gkr777

50. Huang $\mathrm{N}$ et al (2006) Benchmarking sets for molecular docking. J Med Chem 49:6789-6801
51. Srinivasan B et al (2014) Experimental validation of FINDSITEcomb virtual ligand screening results for eight proteins yields novel nanomolar and micromolar binders. J Cheminform 6:16

52. Trott O, Olson AJ (2010) AutoDock Vina: improving the speed and accuracy of docking with a new scoring function, efficient optimization, and multithreading. J Comput Chem 31:455-461

53. Brozell SR et al (2012) Evaluation of DOCK 6 as a pose generation and database enrichment tool. J Comput Aided Mol Des 26:749-773

54. Yang J et al (2013) Protein-ligand binding site recognition using complementary binding-specific substructure comparison and sequence profile alignment. Bioinformatics 29:2588-2595

55. Hajduk PJ, Greer J (2007) A decade of fragment-based drug design: strategic advances and lessons learned. Nat Rev Drug Discov 6:211-219

56. Zhou JZ (ed) (2011) Fragment-based drug design-Springer. Humana Press, New york

57. Hann MM et al (2001) Molecular complexity and its impact on the probability of finding leads for drug discovery. J Chem Inf Comput Sci 41:856-864

58. Carr RAE et al (2005) Fragment-based lead discovery: leads by design. Drug Discov Today 10:987-992

59. Tang GW, Altman RB (2014) Knowledge-based fragment binding predic tion. PLoS Comput Biol 10:e1003589

60. Guedes IA et al (2013) Receptor-ligand molecular docking. Biophys Rev 6:75-87

61. Sharma H et al (2014) Virtual screening reveals allosteric inhibitors of the Toxoplasma gondii thymidylate synthase-dihydrofolate reductase. Bioorg Med Chem Lett 24:1232-1235

62. McGann M (2011) FRED pose prediction and virtual screening accuracy. J Chem Inf Model 51:578-596

63. Irwin JJ et al (2012) ZINC: a free tool to discover chemistry for biology. J Chem Inf Model 52:1757-1768

\section{Publish with ChemistryCentral and every scientist can read your work free of charge \\ "Open access provides opportunities to our colleagues in other parts of the globe, by allowing anyone to view the content free of charge."}

W. Jeffery Hurst, The Hershey Company.

- available free of charge to the entire scientific community

- peer reviewed and published immediately upon acceptance

- cited in PubMed and archived on PubMed Central

- yours - you keep the copyright

Submit your manuscript here:

http://www.chemistrycentral.com/manuscript/ 